\title{
An estimation of p-adic sizes of common zeros of partial derivative polynomials associated with a quartic form
}

\begin{abstract}
In this paper we apply Newton polyhedron technique in estimating the p-adic sizes of common zeros of partial derivative polynomial associated with a quartic polynomial. It is found that the p-adic sizes of a common zeros can be determined explicitly in terms of the $\mathrm{p}$ adic orders of coefficients of dominant terms of polynomial.
\end{abstract}

Keyword: Indicator diagram; Newton polyhedron; p-adic sizes 